\title{
ON SINGLE-LAYER POTENTIALS FOR A CLASS OF PSEUDO-DIFFERENTIAL EQUATIONS RELATED TO LINEAR TRANSFORMATIONS OF A SYMMETRIC $\alpha$-STABLE STOCHASTIC PROCESS
}

\begin{abstract}
In this article an arbitrary invertible linear transformations of a symmetric $\alpha$-stable stochastic process in $d$-dimensional Euclidean space $\mathbb{R}^{d}$ are investigated. The result of such transformation is a Markov process in $\mathbb{R}^{d}$ whose generator is the pseudo-differential operator defined by its symbol $\left(-(Q \xi, \xi)^{\alpha / 2}\right)_{\xi \in \mathbb{R}^{d}}$ with some symmetric positive definite $d \times d$-matrix $Q$ and fixed exponent $\alpha \in(1,2)$. The transition probability density of this process is the fundamental solution of some parabolic pseudo-differential equation. The notion of a single-layer potential for that equation is introduced and its properties are investigated. In particular, an operator is constructed whose role in our consideration is analogous to that the gradient in the classical theory. An analogy to the classical theorem on the jump of the co-normal derivative of the single-layer potential is proved. This result can be applied for solving some boundary-value problems for the parabolic pseudodifferential equations under consideration. For $\alpha=2$, the process under consideration is a linear transformation of Brownian motion, and all the investigated properties of the single-layer potential are well known.
\end{abstract}

Key words and phrases: pseudo-differential equation, single-layer potential, $\alpha$-stable stochastic process, jump theorem.

Vasyl Stefanyk Precarpathian National University, 57 Shevchenka str., 76018, Ivano-Frankivsk, Ukraine

E-mail: mamalygakhrystyna@gmail .com (Mamalyha Kh.V.), myosyp@gmail. com (Osypchuk M.M.)

\section{INTRODUCTION}

Let us consider a symmetric $\alpha$-stable process $\left(x_{0}(t)\right)_{t \geq 0}$ in the $d$-dimensional Euclidean space $\mathbb{R}^{d}$ (we denote by $(\cdot, \cdot)$ the inner product in this space), that is, a Markov process with its transition probability density given by the equality

$$
g_{0}(t, x, y)=\frac{1}{(2 \pi)^{d}} \int_{\mathbb{R}^{d}} e^{i(\xi, x-y)-t|\xi|^{\alpha}} d \xi, \quad t>0, x \in \mathbb{R}^{d}, y \in \mathbb{R}^{d}
$$

where the exponent $\alpha \in(1,2)$ is fixed. The class of all symmetric $\alpha$-stable processes can be obtained from the process $\left(x_{0}(t)\right)_{t \geq 0}$ by multiplying it on some positive constants. More complex processes can be obtained in the following way. 
Let $P$ be some invertible $d \times d$-matrix and $x(t)=P x_{0}(t), t \geq 0$. This process is obviously Markov process and its transition probability density is given by the equality

$$
g(t, x, y)=\frac{1}{(2 \pi)^{d}} \int_{\mathbb{R}^{d}} e^{i(\xi, x-y)-t(Q \xi, \xi)^{\alpha / 2}} d \xi, \quad t>0, x \in \mathbb{R}^{d}, y \in \mathbb{R}^{d},
$$

where $Q=P P^{T}$. It is clear that the process $(x(t))_{t \geq 0}$ is stochastically equivalent to the Markov process $\left(L x_{0}(t)\right)_{t \geq 0}$, where $L$ is some lower triangular matrix which satisfies the equality $Q=$ $L L^{T}$.

The function $g$ is the fundamental solution of the pseudo-differential equation

$$
\frac{\partial u(t, x)}{\partial t}=\mathbf{A} u(t, \cdot)(x), \quad t>0, x \in \mathbb{R}^{d}
$$

where operator $\mathbf{A}$ is a pseudo-differential operator whose symbol is given by the function $\left(-(Q \xi, \xi)^{\alpha / 2}\right)_{\xi \in \mathbb{R}^{d}}$. The operator $\mathbf{A}$ is the generator of Markov process $(x(t))_{t \geq 0}$.

For a given surface $S$, which separates $\mathbb{R}^{d}$ into two open sets $D_{-}$and $D_{+}\left(\mathbb{R}^{d}=D_{-} \cup S \cup\right.$ $\left.D_{+}\right)$and a given continuous function $(\psi(t, x))_{t \geq 0, x \in S}$, we consider a function

$$
v(t, x)=\int_{0}^{t} d \tau \int_{S} g(t-\tau, x, y) \psi(\tau, y) d \sigma_{y}, \quad t>0, x \in \mathbb{R}^{d},
$$

where the inner integral is a surface one. The function $v$ is called a single-layer potential on the surface $S$ with the density $\psi$ for equation (2).

In this article, we determine the existence conditions of the single-layer potential and investigate its properties. The case of $Q=c^{2 / \alpha} I$ ( $c>0$ and $I$ is a unit $d \times d$-matrix) was considered in article [3]. We will use several methods from [3]. In the case of $\alpha=2$, the theory of singlelayer potentials is well-known (see, for example, [2]).

\section{SOME AUXILIARY RESULTS}

\subsection{The function $g$}

The function $g$ defined above by formula (1) is continuous on the domain $t>0, x \in \mathbb{R}^{d}$, $y \in \mathbb{R}^{d}$, and is uniformly continuous on each set of the type $(t, x, y) \in[\tau,+\infty) \times \mathbb{R}^{d} \times \mathbb{R}^{d}$ with $\tau>0$. The following estimations of $g$ and its derivatives are known (see [1, Ch.4]):

$$
\begin{aligned}
& \left|\mathbf{D}^{k} g(t, \cdot, y)(x)\right| \leq N_{k} \frac{t}{\left(t^{1 / \alpha}+|y-x|\right)^{d+\alpha+k}}, \quad t>0, x \in \mathbb{R}^{d}, y \in \mathbb{R}^{d} ; \\
& \left|\mathbf{D}^{\varkappa} g(t, \cdot, y)(x)\right| \leq \tilde{N}_{\varkappa} \frac{1}{\left(t^{1 / \alpha}+|y-x|\right)^{d+\varkappa}}, \quad t>0, x \in \mathbb{R}^{d}, y \in \mathbb{R}^{d} .
\end{aligned}
$$

Here $\mathbf{D}^{k}$ means a differential operator of the order $k(k=0,1,2, \ldots), \mathbf{D}^{\varkappa}$ means a pseudodifferential operator with a homogeneous symbol $\left(p_{\varkappa}(\xi)\right)_{\xi \in \mathbb{R}^{d}}$ of the order $\varkappa$ which has all derivatives of the orders $l<M$ with some $M \geq 2 d+\varkappa+\alpha+1$ and $\left|p_{\varkappa}^{(l)}(\xi)\right| \leq C_{M}|\xi|^{\varkappa-1}$ with some constant $C_{M} \geq 0$ for all $\xi \neq 0$, and $N_{k}$ and $\tilde{N}_{\varkappa}$ are some positive constants. 


\subsection{The operator $\mathrm{A}$}

An action of the operator A defined in Introduction on a smooth (with at least Lipschitz continuous gradient) and bounded together with its derivatives function $\varphi(x)_{x \in \mathbb{R}^{d}}$ is given by the expression

$$
\mathbf{A} \varphi(x)=\frac{q_{\alpha}}{(\operatorname{det} Q)^{1 / 2}} \int_{\mathbb{R}^{d}}(\varphi(x+y)-\varphi(x)-(\nabla \varphi(x), y))\left(Q^{-1} y, y\right)^{-(d+\alpha) / 2} d y,
$$

where

$$
q_{\alpha}=\frac{\alpha \Gamma((3-\alpha) / 2) \Gamma((d+\alpha) / 2)}{\pi^{(d+1) / 2} \Gamma(2-\alpha)} .
$$

The value of the constant $q_{\alpha}$ can be obtained by applying the operator $\mathbf{A}$ to the function $\varphi_{\xi}(x)=$ $e^{i(\xi, x)}, x \in \mathbb{R}^{d}$ with some fixed $\xi \in \mathbb{R}^{d}$.

\subsection{An operator $B$}

Let us introduce the operator $\mathbf{B}$ using its symbol $\left(i(Q \xi, \xi)^{\alpha / 2-1} \xi\right)_{\xi \in \mathbb{R}^{d}}$. Some simple calculations lead us to the relation $\mathbf{A}=(\nabla, Q \mathbf{B})$. The action of the operator $\mathbf{B}$ on a bounded Lipschitz continuous function $(\varphi(x))_{x \in \mathbb{R}^{d}}$ is defined by the following formula

$$
\mathbf{B} \varphi(x)=\frac{q_{\alpha}}{\alpha(\operatorname{det} Q)^{1 / 2}} \int_{\mathbb{R}^{d}}(\varphi(x+y)-\varphi(x))\left(Q^{-1} y, y\right)^{-(d-\alpha) / 2} Q^{-1} y d y,
$$

where $q_{\alpha}$ has the above meaning.

Let $v$ be some fixed ort in $\mathbb{R}^{d}$. Consider the operator $\mathbf{B}_{v}=2(Q v, \mathbf{B})$. We denote the result of its action on the function $g$ with respect to the second argument by $g_{v}(t, x, y)$. Using representation (1) of the function $g$ and the integration by parts, it is easy to obtain the relation

$$
g_{v}(t, x, y)=\frac{2}{\alpha} \frac{(y-x, v)}{t} g(t, x, y), \quad t>0, x \in \mathbb{R}^{d}, y \in \mathbb{R}^{d} .
$$

\subsection{A surface of the class $H^{1+\gamma}$}

Let some surface $S$ in $\mathbb{R}^{d}$ (a manifold of dimension $d-1$ ) divide the set $\mathbb{R}^{d}$ into two open sets: outer $D_{+}$and inner $D_{-}$(i.e., $\mathbb{R}^{d}=D_{-} \cup S \cup D_{+}$). Suppose that this surface has a tangent hyperplane at each point $x \in S$. We will denote $v(x)$ the unit vector of the outer normal to the surface $S$ at the point $x \in S$. Choose the point $x \in S$ and consider a local orthogonal coordinate system with the origin at this point, such that $v(x)$ is the ort of its last axis. Assume the surface $S$ is such that for some $\delta>0$ each part $S_{\delta}(x)=S \cap B_{\delta}(x), x \in S$, of the surface $S$ (here $B_{\delta}(x)$ is a ball with the radius $\delta>0$ and the center at the point $x$ ) can be described in the mentioned above local coordinate system by the equation $y_{d}=F_{x}\left(y_{1}, \ldots, y_{d-1}\right)$ with a single-valued function $F_{x}$.

The bounded closed surface $S$ belongs to the class $H^{1+\gamma}$ if the function $F_{x}$ has all partial derivatives $\frac{\partial F_{x}}{\partial y_{k}}, k=1,2, \ldots, d-1$, satisfy Hölder's condition with a power $\gamma \in(0 ; 1)$ and the constant does not dependent on $x$.

Among the properties of the surface $S$ which belongs to the class $H^{1+\gamma}$ we will use the following one (see [2, Ch.5]): there are some positive numbers $\delta_{0}$ and $r_{0}$, and a finite set of points $x_{1}, x_{2}, \ldots, x_{m}$ on the surface $S$, such that $S \backslash S_{r_{0} / 2}(x) \subset \cup_{k \in I_{x}} S_{r_{0} / 2}\left(x_{k}\right)$ for each $x \in S$, and $\min _{k \in I_{x}} \inf _{y \in S_{r_{0} / 2}\left(x_{k}\right)}|y-x| \geq \delta_{0}$, where $I_{x}$ is some subset of the indices $\{1,2, \ldots, m\}$. 


\section{A SINGLE-LAYER POTENTIAL}

\subsection{Existence conditions}

Let $S$ be a bounded closed surface of the class $H^{1+\gamma}$ with some $\gamma \in(0 ; 1)$. Consider some continuous function $(\psi(t, x))_{t \geq 0, x \in S}$ and define the function $(v(t, x))_{t \geq 0, x \in \mathbb{R}^{d}}$ by the following equality

$$
v(t, x)=\int_{0}^{t} d \tau \int_{S} g(t-\tau, x, y) \psi(\tau, y) d \sigma_{y}, \quad t>0, x \in \mathbb{R}^{d}, y \in \mathbb{R}^{d},
$$

where an inner integral is a surface one. This function is called a single-layer potential on the surface $S$ with the density $\psi$. The following statement contains the conditions under which a single-layer potential is well defined.

Lemma 1. Let $S$ be a bounded closed surface of the class $H^{1+\gamma}$ with some $\gamma \in(0 ; 1)$ and $(\psi(t, x))_{t \geq 0, x \in S}$ be a continuous function, which satisfies the inequality $|\psi(t, x)| \leq C_{T} t^{-\beta}$ in each set of $(t ; x) \in(0 ; T] \times S$ with some constants $\beta<1$ and $C_{T}>0$ (the last one can depend on $T>0$ ). Then, the single-layer potential (6) is finite for all $t>0$ and $x \in \mathbb{R}^{d}$.

Proof. Estimation (3) with $k=0$ and the fact that (see [3])

$$
\int_{S} \frac{d \sigma_{y}}{\left(t^{1 / \alpha}+|y-x|\right)^{d+\alpha}} \leq K t^{-1-1 / \alpha}, t>0, x \in \mathbb{R}^{d}
$$

with some constant $K>0$ imply to the inequality

$$
|v(t, x)| \leq K N_{0} C_{T} \int_{0}^{t} \frac{d \tau}{\tau^{\beta}(t-\tau)^{1 / \alpha}}=K N_{0} C_{T} B(1-\beta, 1-1 / \alpha) t^{1-\beta-1 / \alpha}
$$

for all $t \in(0 ; T], x \in \mathbb{R}^{d}$ and each $T>0$.

\subsection{Properties of the single-layer potential}

Classically (when $\alpha=2$ ), a single-layer potential satisfies the appropriate parabolic differential equation in the domain $(0 ;+\infty) \times\left(\mathbb{R}^{d} \backslash S\right)$ (see [2, Ch.5] ). Let us prove an analogous statement in our case $(1<\alpha<2)$.

Theorem 1. Let $S$ be a bounded closed surface of the class $H^{1+\gamma}$ with some $\gamma \in(0 ; 1)$, and $(\psi(t, x))_{t \geq 0, x \in S}$ be a continuous function satisfying the inequality $|\psi(t, x)| \leq C_{T} t^{-\beta}$ in each set of $(t ; x) \in(0 ; T] \times S$ with some constants $\beta<1$ and $C_{T}>0$ (the last one can depend on $T>0$ ). Then the single-layer potential (3) satisfies the equation

$$
\frac{\partial v(t, x)}{\partial t}=\boldsymbol{A} v(t, \cdot)(x), \quad t>0, x \in \mathbb{R}^{d}
$$

in the domain $(t ; x) \in(0 ; \infty) \times\left(\mathbb{R}^{d} \backslash S\right)$.

Proof. It has already been mentioned that the function $g$ is the fundamental solution of equation (2), and therefore, for all $t>0, x \in \mathbb{R}^{d}, y \in \mathbb{R}^{d}$ the equality $\frac{\partial g(t, x, y)}{\partial t}=\mathbf{A} g(t, \cdot, y)(x)$ holds true. So, we only have to prove that the operator $\mathbf{A}$ with respect to the variable $x$ can be moved under the integral symbol in the right-hand part of (6) and the equality 


$$
\lim _{\varepsilon \rightarrow 0+} \int_{S} g(\varepsilon, x, y) \psi(t, y) d \sigma_{y}=0,
$$

holds true for $t>0, x \in \mathbb{R}^{d} \backslash S$. The last is due to estimation (3) with $k=0$ and the inequality

$$
\left|\int_{S} g(\varepsilon, x, y) \psi(t, y) d \sigma_{y}\right| \leq N_{0} \varepsilon \frac{|S|}{(\rho(x, S))^{d+\alpha}} C_{T} t^{-\beta},
$$

where $|S|$ is the area of the surface $S$, and $\rho(x, S)$ is the distance from the point $x$ to the surface $S$.

Next, we will take presentation (4) of the operator A and prove the possibility to change the order of integrating in the integral

$$
\int_{0}^{t} d \tau \int_{S} \psi(\tau, y) d \sigma_{y} \int_{\mathbb{R}^{d}} \frac{g(t-\tau, x+z, y)-g(t-\tau, x, y)-(\nabla g(t-\tau, \cdot, y)(x), z)}{\left(Q^{-1} z, z\right)^{(d+\alpha) / 2}} d z .
$$

Take into account that the following inequalities $\frac{1}{M}|z|^{2} \leq\left(Q^{-1} z, z\right) \leq M|z|^{2}$ hold true with some constant $M>0$ for all $x \in \mathbb{R}^{d}$. Divide the last integral into the sum of two integrals $I_{1}$ and $I_{2}$ taken from the same function: the first of them is by $(0 ; t) \in S \times B_{\varepsilon}$, and another is by $(0 ; t) \in S \times\left(\mathbb{R}^{d} \backslash B_{\varepsilon}\right)$, where $B_{\varepsilon}$ is a ball of some small enough radius $\varepsilon>0$ centered at the origin.

Since for $0<\tau<t, x \in \mathbb{R}^{d}, y \in S, z \in B_{\varepsilon}$ the following equality

$$
g(t-\tau, x+z, y)-g(t-\tau, x, y)-(\nabla g(t-\tau, \cdot, y)(x), z)=\frac{1}{2} \sum_{i, j=1}^{d} \frac{\partial^{2} g(t-\tau, x+\theta z, y)}{\partial x_{i} \partial x_{j}} z_{i} z_{j}
$$

is true, where $\theta=\theta(\tau ; y) \in(0 ; 1)$, the absolute value of the integrand in $I_{1}$ is estimated by the expression

$$
C_{t} \tau^{-\beta} \frac{1}{2} d^{2} N_{2} \frac{t-\tau}{\left((t-\tau)^{1 / \alpha}+|y-x-\theta z|\right)^{d+\alpha+2}}|z|^{2} M^{(d+\alpha) / 2}|z|^{-d-\alpha}
$$

where estimation (3) for $k=2$ is used. Take a sufficiently small $\varepsilon>0$ such that the inequality inf $_{y \in S, z \in B_{\varepsilon}, \theta \in(0,1)}|y-x-\theta z|=\rho_{0}>0$ holds true. Therefore, $I_{1}$ is absolutely convergent.

The absolute value of the integrand in $I_{2}$ is estimated by the expression (estimation (3) is also taken into account)

$$
\begin{aligned}
& C_{t} \tau^{-\beta} M^{(d+\alpha) / 2}|z|^{-d-\alpha}\left(\frac{N_{0}(t-\tau)}{\left((t-\tau)^{1 / \alpha}+|y-x-z|\right)^{d+\alpha}}\right.+\frac{N_{0}(t-\tau)}{\left((t-\tau)^{1 / \alpha}+|y-x|\right)^{d+\alpha}} \\
&\left.+\frac{N_{1}(t-\tau)}{\left((t-\tau)^{1 / \alpha}+|y-x|\right)^{d+\alpha+1}}|z|\right) \\
& \leq C_{t} \tau^{-\beta} M^{(d+\alpha) / 2}|z|^{-d-\alpha}\left(\frac{N_{0}(t-\tau)}{\left((t-\tau)^{1 / \alpha}+|y-x-z|\right)^{d+\alpha}}+\frac{N_{0}(t-\tau)}{(\rho(x, S))^{d+\alpha}}+\frac{N_{1}(t-\tau)}{(\rho(x, S))^{d+\alpha+1}}|z|\right) .
\end{aligned}
$$

Observe that the second and the third terms in this expression are integrable. Consider the integral of the first term and change the variable $z$ into $u$ using the equality $y-x-z=$ $(t-\tau)^{1 / \alpha} u$. We have got

$$
N_{0} C_{t} M^{(d+\alpha) / 2} \int_{0}^{t} \frac{(t-\tau)^{1+d / \alpha}}{\tau^{\beta}} d \tau \int_{S} d \sigma_{y} \int_{D(\tau, y)}\left|y-x-(t-\tau)^{1 / \alpha} u\right|^{-d-\alpha}(1+|u|)^{-d-\alpha} d u,
$$

where $D(\tau, y)=\left\{u \in \mathbb{R}^{d}:\left|y-x-(t-\tau)^{1 / \alpha} u\right|>\varepsilon\right\}$. Surely, this integral converges, and for completing the proof of the theorem, we have to use the Fubini's theorem. 


\subsection{The jump theorem}

In a classical theory (with $\alpha=2$ ) of a single-layer potential the jump theorem takes an essential place. It is the theorem on the jump of the co-normal derivative of a single-layer potential. This section is devoted to an analogue of that theorem in our situation $(1<\alpha<2)$.

Lemma 2. Let the surface $S$ and the function $(\psi(t, x))_{t \geq 0, x \in S}$ satisfy the conditions of Theorem 1. Then for each $t>0$ and $x \in S$ the following integral

$$
\int_{0}^{t} d \tau \int_{S} \mathbf{B}_{v(x)} g(t-\tau, \cdot, y)(x) \psi(\tau, y) d \sigma_{y}
$$

is finite.

Proof. By equality (5), we can rewrite integral (7) in the following form

$$
\frac{2}{\alpha} \int_{0}^{t} \frac{d \tau}{t-\tau} \int_{S}(y-x, v(x)) g(t-\tau, x, y) \psi(\tau, y) d \sigma_{y} .
$$

Taking into account estimation (3) and the properties of the surface $S$ (see Section 1.1), we can obtain for $\tau<t, x \in S$, the inequality

$$
\begin{aligned}
\left|\int_{S}(y-x, v(x)) g(t-\tau, x, y) \psi(\tau, y) d \sigma_{y}\right| & \leq C_{t} \tau^{-\beta} K\left((t-\tau)^{\gamma / 2}+\left((t-\tau)^{1 / 2}+\delta_{0}\right)^{-d-\alpha+1}(t-\tau)\right) \\
& \leq \text { const }_{t} \tau^{-\beta}\left((t-\tau)^{\gamma / 2}+(t-\tau)\right),
\end{aligned}
$$

where $K>0$ is some constant and const $t_{t}$ is some positive constant, which probably depends on $t$. Hence the statement of the lemma is proved.

Remark 1. Integral (7) is called a direct value of the action result of the operator $\mathbf{B}_{v(x)}, x \in S$ on single-layer potential (6) at the point $x \in S$. We will denote it by $\mathbf{B}_{v(x)}^{(d v)} v(t, \cdot)(x)$.

The next statement is the jump theorem mentioned above.

Theorem 2. Let $S$ be a bounded closed surface of the class $H^{1+\gamma}$ with some $\gamma \in(0 ; 1)$ in $\mathbb{R}^{d}$, and $(\psi(t, x))_{t \geq 0, x \in S}$ be a continuous function satisfying the inequality $|\psi(t, x)| \leq C_{T} t^{-\beta}$, $0<t \leq T, x \in S$ with some constants $\beta<1$ and $C_{T}>0$ (the last one can depend on $T$ ) for each $T>0$. Then for each $t \geq 0, x \in S$ the following equality

$$
\lim _{y \rightarrow x \pm} \mathbf{B}_{v(x)} v(t, \cdot)(y)=\mp \psi(t, x)+\mathbf{B}_{v(x)}^{(d v)} v(t, \cdot)(x),
$$

holds true, where $y \rightarrow x \pm$ means that $y$ approaches $x$ staying in some closed bounded cone $\mathcal{K} \subset \mathbb{R}^{d}$ with the vertex at the point $x$ and $\mathcal{K} \subset D_{ \pm} \cup\{x\}$.

Proof. Similar to the classic case it is sufficient to consider only the case of $y=x+\delta v(x)$ and $\delta \rightarrow 0 \pm$. Therefore, taking into account formula (5) we will obtain

$$
\begin{aligned}
\mathbf{B}_{v(x)} v(t, \cdot)(y) & =\frac{2}{\alpha} \int_{0}^{t} \frac{d \tau}{t-\tau} \int_{S}(z-x, v(x)) g(t-\tau, y, z) \psi(\tau, z) d \sigma_{z} \\
& -\delta \frac{2}{\alpha} \int_{0}^{t} \frac{d \tau}{t-\tau} \int_{S} g(t-\tau, y, z) \psi(\tau, z) d \sigma_{z} \\
=\mathbf{B}_{v(x)}^{(d v)} v(t, \cdot)(x) & +\frac{2}{\alpha} \int_{0}^{t} \frac{d \tau}{t-\tau} \int_{S}(z-x, v(x))(g(t-\tau, y, z)-g(t-\tau, x, z)) \psi(\tau, z) d \sigma_{z} \\
& -\delta \frac{2}{\alpha} \int_{0}^{t} \frac{d \tau}{t-\tau} \int_{S} g(t-\tau, y, z) \psi(\tau, z) d \sigma_{z} .
\end{aligned}
$$


Denote the integrals on the right-hand side of this equality by $I_{1}$ and $I_{2}$ accordingly.

First, let us prove that $\lim _{\delta \rightarrow 0} I_{1}=0$. In order to get this proof, rewrite $I_{1}$ in the form of the sum of the following expressions

$$
\begin{gathered}
J_{1}^{(1)}=\frac{2}{\alpha} \int_{0}^{t-\rho} \frac{d \tau}{t-\tau} \int_{S}(z-x, v(x))(g(t-\tau, x+\delta v(x), z)-g(t-\tau, x, z)) \psi(\tau, z) d \sigma_{z}, \\
J_{2}^{(1)}=\frac{2}{\alpha} \int_{t-\rho}^{t} \frac{d \tau}{t-\tau} \int_{S_{r_{0} / 2}(x)}(z-x, v(x))(g(t-\tau, x+\delta v(x), z)-g(t-\tau, x, z)) \psi(\tau, z) d \sigma_{z}, \\
J_{3}^{(1)}=\frac{2}{\alpha} \int_{t-\rho}^{t} \frac{d \tau}{t-\tau} \int_{S \backslash S_{r_{0} / 2}(x)}(z-x, v(x))(g(t-\tau, x+\delta v(x), z)-g(t-\tau, x, z)) \psi(\tau, z) d \sigma_{z},
\end{gathered}
$$

where $0<\rho<t$ is some constant ( $t$ is fixed), which should be chosen. We estimate each of these expressions. Taking into account the properties of the surface $S$, we can obtain $\mid(z-$ $x, v(x))|\leq| z-\left.x\right|^{1+\gamma}$ for $z \in S_{r_{0 / 2}}(x)$. As a result, we have

$$
\begin{aligned}
\left|J_{2}^{(1)}\right| \leq C_{t} \frac{2}{\alpha} \int_{t-\rho}^{t} \frac{d \tau}{\tau^{\beta}} \int_{S_{r_{0 / 2}}(x)} \frac{|z-x|^{1+\gamma}}{\left((t-\tau)^{1 / \alpha}+|z-x-\delta v(x)|\right)^{d+\alpha}} d \sigma_{z} \\
\quad+C_{t} \frac{2}{\alpha} \int_{t-\rho}^{t} \frac{d \tau}{\tau^{\beta}} \int_{S_{r_{0 / 2}}(x)} \frac{|z-x|^{1+\gamma}}{\left((t-\tau)^{1 / \alpha}+|z-x|\right)^{d+\alpha}} d \sigma_{z} .
\end{aligned}
$$

Let $z$ be the orthogonal projection of the point $z \in S_{r_{0 / 2}}(x)$ on the tangent hyperplane to $S$ at the point $x$. Hence, taking into account the inequalities $|z-x| \geq|\tilde{z}-x|,|z-x-\delta v(x)| \geq|\tilde{z}-x|$, $|z-x| \geq$ const $|\tilde{z}-x|$, where const is some positive constant that does not depend on the point $x$ (see $[2$, Ch.5]), we will obtain to the inequalities

$$
\left|J_{2}^{(1)}\right| \leq \hat{C}_{t} \frac{2}{\alpha} \int_{t-\rho}^{t} \frac{d \tau}{\tau^{\beta}} \int_{\triangle K_{r_{0} / 2}(x)} \frac{|\tilde{z}| d \tilde{z}}{\left((t-\tau)^{1 / \alpha}+|\tilde{z}|\right)^{d+\alpha}} d \sigma_{z} \leq \frac{\tilde{C}_{t}}{(t-\rho)^{\beta}} \rho^{\gamma / 2}
$$

where $\hat{C}_{t}, \tilde{C}_{t}$ are positive constants that probably depend on $t$, and $\triangle_{r_{0 / 2}}(x) \subset \mathbb{R}^{d-1}$ is some bounded set. It means that $\triangle_{r_{0 / 2}}(x)$ is the orthogonal projection of $S_{r_{0 / 2}}(x)$ on the tangent hyperplane to $S$ at the point $x \in S$ in the coordinate system of this hyperplane.

Next, we will estimate $J_{3}^{(1)}$ :

$$
\begin{aligned}
\left|J_{3}^{(1)}\right| \leq C_{t} & \int_{t-\rho}^{t} \frac{d \tau}{\tau^{\beta}} \int_{S \backslash S_{r_{0 / 2}}(x)} \frac{|z-x|}{\left((t-\tau)^{1 / \alpha}+|z-x-\delta v(x)|\right)^{d+\alpha}} d \sigma_{z} \\
& +C_{t} \int_{t-\rho}^{t} \frac{d \tau}{\tau^{\beta}} \int_{S \backslash S_{r_{0} / 2}(x)} \frac{|z-x|}{\left((t-\tau)^{1 / \alpha}+|z-x|\right)^{d+\alpha}} d \sigma_{z} .
\end{aligned}
$$

Taking into account that for $z \in S \backslash S_{r_{0 / 2}}(x)$ the inequalities $|z-x| \geq \delta_{0},|z-x-\delta v(x)| \geq$ $|z-x|-|\delta| \geq \delta_{0}-|\delta|$ are true (choose $\delta$ to be the one that $|\delta|<\delta_{0}$ ), we will have

$$
\left|J_{3}{ }^{(1)}\right| \leq \hat{C}_{t}\left(\delta_{0}-|\delta|\right)^{-d-\alpha} \int_{t-\rho}^{t} \frac{d \tau}{\tau^{\beta}} .
$$

Thus, the sum $J_{2}^{(1)}+J_{3}^{(1)}$ can be made as small as we want by choosing $\rho>0$. 
Now, consider $J_{1}^{(1)}$. Since the function $g(t-\tau, x, z)$ is uniformly continuous in the sets of the type $(\tau, x, z) \in[0 ; t-\rho] \times K_{1} \times K_{2}$, where $K_{1}$ and $K_{2}$ are some compacta in $\mathbb{R}^{d}$, and taking into account the integrability of the function $\psi$ on $[0 ; t-\rho] \times S$ and the boundary of the function $(z-x, v(x))$ as the function of $z$ on $S$, we will obtain that $\lim _{\delta \rightarrow 0} J_{1}^{(1)}=0$. Therefore, $I_{1} \rightarrow 0$ as $\delta \rightarrow 0$.

Now, consider the behavior of $I_{2}$ as $\delta \rightarrow 0$. Put $I_{2}$ in the form of the sum of the following expressions

$$
\begin{gathered}
J_{1}^{(2)}=\frac{2}{\alpha} \delta \psi(t, x) \int_{t-\rho}^{t} \frac{d \tau}{t-\tau} \int_{S_{\varepsilon}(x)} g(t-\tau, x+\delta v(x), z) d \sigma_{z} \\
J_{2}^{(2)}=\frac{2}{\alpha} \delta \int_{t-\rho}^{t} \frac{d \tau}{t-\tau} \int_{S_{\varepsilon}(x)} g(t-\tau, x+\delta v(x), z)(\psi(\tau, z)-\psi(t, x)) d \sigma_{z} \\
J_{3}^{(2)}=\frac{2}{\alpha} \delta \int_{0}^{t-\rho} \frac{d \tau}{t-\tau} \int_{S_{\varepsilon}(x)} g(t-\tau, x+\delta v(x), z) \psi(\tau, z) d \sigma_{z} \\
J_{4}^{(2)}=\frac{2}{\alpha} \delta \int_{0}^{t} \frac{d \tau}{t-\tau} \int_{S \backslash S_{\varepsilon}(x)} g(t-\tau, x+\delta v(x), z) \psi(\tau, z) d \sigma_{z}
\end{gathered}
$$

where $\rho>0, \varepsilon>0$ are rather small constants.

Let us estimate each of these terms. We start with the last one. Taking into account the properties of the surface $S$, there are numbers $l_{0}$ (natural) and $p_{0}>0$, such that we can find points $x_{k} \in S \backslash S_{\varepsilon}(x), k=1,2 \ldots, l_{0}$ that $S \backslash S_{\varepsilon}(x) \subset \cup_{k=1}^{l_{0}} S_{r_{0 / 2}}\left(x_{k}\right)$ and $\inf _{|\xi| \leq|\delta|} \inf _{z \in S_{r_{0 / 2}}\left(x_{k}\right)}|z-x-\zeta v(x)| \geq p_{0}$. Then estimation (3) implies

$$
\left|J_{4}^{(2)}\right| \leq \frac{2}{\alpha} C_{t} N_{0}|S| l_{0}|\delta| \int_{0}^{t} \tau^{-\beta}\left((t-\tau)^{1 / \alpha}+p_{0}\right)^{-d-\alpha} d \tau \rightarrow 0, \quad \delta \rightarrow 0 .
$$

Similarly, using inequality (3) we will get

$$
\begin{aligned}
\left|J_{3}^{(2)}\right| & \leq \frac{2}{\alpha}|\delta| C_{t} N_{0} \int_{0}^{t-\rho} \frac{d \tau}{t^{\beta}} \int_{S_{\varepsilon}(x)} \frac{d \sigma_{z}}{\left((t-\tau)^{1 / \alpha}+|z-x-\delta v(x)|\right)^{d+\alpha}} \\
& \leq \frac{2}{\alpha}|\delta| C_{t} N_{0}|S| \int_{0}^{t-\rho} \frac{d \tau}{t^{\beta}(t-\tau)^{1+d / \alpha}} \rightarrow 0, \quad \delta \rightarrow 0 .
\end{aligned}
$$

Here $|S|$ means the area of the surface $S$ like mentioned above.

Now, prove the existence of a limit of $J_{1}^{(2)}$ as $\delta \rightarrow 0$. By the way, it means that by choosing $\rho>0$ and $\varepsilon>0$ the term $J_{2}^{(2)}$ can be made as small as you like. It is sufficiently to note that the function $\psi$ is uniformly continuous on the set $[t-\rho ; t] \times S_{\varepsilon}(x)$. Denote the tangent hyperplane to the surface $S$ at the point $x \in S$ by $\Pi_{x}$, and consider

$$
R=\frac{2}{\alpha} \delta \int_{t-\rho}^{t} \frac{d \tau}{t-\tau} \int_{\Pi_{x}} g(t-\tau, x+\delta v(x), z) d \sigma_{z} .
$$

To prove that $\lim _{\delta \rightarrow 0}\left(J_{1}^{(2)}-\psi(t, x) R\right)=0$, consider

$$
\begin{aligned}
& \delta \int_{0}^{\rho} \frac{d \tau}{\tau}\left(\int_{S_{\varepsilon}(x)}-\int_{\Pi_{x}}\right) g(\tau, x+\delta \nu(x), z) d \sigma_{z} \\
&=\delta \int_{0}^{\rho} \frac{d \tau}{\tau}\left(\int_{S_{\varepsilon}(x)}-\int_{\Pi_{x}}\right) g(\tau, x+\delta \nu(x), z) d \sigma_{z} \\
& \quad \quad-\delta \int_{0}^{\rho} \frac{d \tau}{\tau} \int_{\Pi_{x} \backslash \Pi_{\varepsilon}(x)} g(\tau, x+\delta v(x), z) d \sigma_{z} \\
&=J^{\prime}+J^{\prime \prime}
\end{aligned}
$$


where $\Pi_{\mathcal{E}}(x)$ is the orthogonal projection $S_{\varepsilon}(x)$ on $\Pi_{x}$. Taking into account the properties of the surface $S$, it is easy to see that there is a constant $\theta>0$ such that for all $z \in \Pi_{x} \backslash \Pi_{\mathcal{E}}(x)$ the inequality $|z| \geq \theta$ is true. Therefore the following estimation

$$
\left|J^{\prime \prime}\right| \leq C|\delta| \rho \int_{\theta}^{\infty} \frac{r^{d-2} d r}{\left(\delta^{2}+r^{2}\right)^{(d+\alpha) / 2}}=C \rho \int_{\theta /|\delta|}^{\infty} \frac{r^{d-2}}{\left(1+r^{2}\right)^{(d+\alpha) / 2}} \frac{d r}{|\delta|^{\alpha}}
$$

holds true with some constant $C>0$. As a result, taking L'Hôpital's rule, we will obtain that $J^{\prime \prime} \rightarrow 0$ as $\delta \rightarrow 0$.

In order to estimate $J^{\prime}$ let us transfer it to the local coordinate system with its origin at the point $x$ and the vector $v(x)$ as the ort of its last axis. We have

$$
\begin{gathered}
S_{\varepsilon}(x)=\left\{u \in \mathbb{R}^{d}: u^{d}=F_{x}\left(u^{<d>}\right), u^{<d>} \in D_{\varepsilon}(x) \subset \mathbb{R}^{d-1}\right\}, \\
\Pi_{\varepsilon}(x)=\left\{u \in \mathbb{R}^{d}: u^{d}=0, u^{<d>} \in D_{\varepsilon}(x) \subset \mathbb{R}^{d-1}\right\},
\end{gathered}
$$

where $D_{\varepsilon}(x)$ is some bounded closed set depended only on properties of the surface $S, u^{<d>}$ is the vector $\left(u_{1}, u_{2}, \ldots, u_{d-1}\right)$, and $F_{x}$ is some single-valued function with Hölder continuous gradient (see Section 1.1) with power $\gamma \in(0 ; 1)$. Talking into account inequality (3), it is not difficult to state that

$$
\begin{aligned}
\left|J^{\prime}\right| & \leq K|\delta| \int_{0}^{\rho} \frac{d \tau}{\tau} \int_{D_{\varepsilon}(x)} \frac{\tau|u|^{\gamma}\left(1+|u|^{\gamma}\right) d u}{\left(\tau^{1 / \alpha}+k \sqrt{|u|^{2}+\delta^{2}}\right)^{d+\alpha}} \\
& \leq \hat{K}|\delta| \int_{0}^{\rho} d \tau \int_{0}^{\varepsilon_{0}} \frac{r^{d-2+\gamma} d r}{\left(\tau^{1 / \alpha}+k \sqrt{r^{2}+\delta^{2}}\right)^{d+\alpha}}
\end{aligned}
$$

where $K>0, \hat{K}>0, k>0, \varepsilon_{0}>0$ are some constants. Changing the order of integration in the last integral and taking into account the equality $\int_{0}^{\infty} \frac{d \tau}{\left(\tau^{1 / \alpha}+a\right)^{d+\alpha}}=\alpha B(d, \alpha) a^{-d}$ that is correct for all $a>0$, we will obtain the estimation

$$
\left|J^{\prime}\right| \leq \tilde{K}|\delta| \int_{0}^{\varepsilon_{0}} \frac{r^{d-2+\gamma} d r}{\left(\sqrt{r^{2}+\delta^{2}}\right)^{d}} \leq \tilde{K} \int_{0}^{\infty} \frac{r^{d-2+\gamma} d r}{\left(\sqrt{r^{2}+1}\right)^{d}}|\delta|^{\delta}
$$

with some constant $\tilde{K}>0$. Therefore $J^{\prime} \rightarrow 0$ as $\delta \rightarrow 0$ and $\lim _{\delta \rightarrow 0}\left(J_{1}^{(2)}-\psi(t, x) R\right)=0$. Thus, we have to prove the existence of $\lim _{\delta \rightarrow 0 \pm} R$ and to find it. In order to prove this we will take the equality proved in [3] ( $\hat{v}$ is a fixed ort in $\mathbb{R}^{d}$ )

$$
\int_{\Pi} h_{d}(x+\lambda \hat{v}) d \sigma_{x}=\frac{1}{\pi} \int_{0}^{\infty} e^{-c r^{\alpha}} \cos \lambda r d r
$$

where $\lambda \in \mathbb{R}^{d}, \Pi=\left\{x \in \mathbb{R}^{d}:(x, \hat{v})=0\right\}$ and $h_{d}(x)=(2 \pi)^{-d} \int_{\mathbb{R}^{d}} e^{i(x ; \xi)-c|\xi|^{\alpha}} d \xi, x \in \mathbb{R}^{d}$. Therefore, after simple transformations related with the changing of the variables in the surface integral, we will obtain the equality

$$
I=\int_{\Pi_{x}} g(t, x+\delta v(x), y) d \sigma_{y}=t^{-1 / \alpha} \int_{\Pi} h_{d}\left(z-\delta t^{-1 / \alpha} \hat{v}\right) d \sigma_{z}
$$

where $\Pi=\left\{z \in \mathbb{R}^{d}:(z, \hat{v})=0\right\}, \hat{v}$ is some ort in $\mathbb{R}^{d}$. Equality (8) implies 


$$
I=t^{-1 / \alpha} \frac{1}{\pi} \int_{0}^{\infty} e^{-c r^{\alpha}} \cos r \delta t^{-1 / \alpha} d r=\frac{1}{\pi} \int_{0}^{\infty} e^{-c t r^{\alpha}} \cos r \delta d r
$$

Thus,

$$
R=\frac{2}{\alpha \pi} \delta \int_{t-\rho}^{t} \frac{d \tau}{t-\tau} \int_{0}^{\infty} e^{-c(t-\tau) r^{\alpha}} \cos \delta r d r
$$

By changing the order of integration in this integral, we obtain the equality

$$
R=\operatorname{sign} \delta-\frac{2}{\pi} \int_{0}^{\infty} e^{-c \rho r^{\alpha}} \frac{\sin \delta r}{r} d r
$$

and, therefore, we have that $\lim _{\delta \rightarrow 0 \pm} R= \pm 1$. Hence, $\lim _{\delta \rightarrow 0 \pm} J_{1}^{(2)}= \pm \psi(t, x), t>0, x \in S$ and the theorem has been proved.

\subsection{The single-layer potential with a hyperplane as a carrier}

Let $S$ be a hyperplane defined by the equation $(x, v)=r$, where $v \in \mathbb{R}^{d}$ is some unit vector, and $r \in \mathbb{R}$ is a fixed real number. Let the function $(\psi(t, x))_{t \geq 0, x \in S}$ be continuous as mentioned above, and the inequality $|\psi(t, x)| \leq C_{T} t^{-\beta}$ be true for all $0<t \leq T, x \in S$ and each $T>0$. Here the constant $C_{T}>0$ probably depends on $T$ and $\beta<1$. Taking into account inequality (3), we obtain the estimation $\int_{S} g(t, x, y) d \sigma_{y} \leq K t^{-1 / \alpha}, t>0, x \in \mathbb{R}^{d}$ and for the fixed $x \in \mathbb{R}^{d} \backslash S$ we have $\int_{S} g(t, x, y) d \sigma_{y} \leq M t, t>0$, where $K>0$ is some constant, and $M>0$ is the constant depending on $x$. This, is analogous to the previous, one can state that the statements of Lemma 1 and Theorem 1 are true in this case ( $S$ is a hyperplane) as well.

Furthermore, formula (5) shows that $g_{v}(t, x, y)=0$ for all $t>0, x \in \mathbb{R}^{d}, y \in \mathbb{R}^{d}$. Therefore, the equality $\mathbf{B}_{v(x)}^{(d v)} v(t, \cdot)(x)=0, \quad t>0, x \in S$ holds true. Thus, an analogue of Theorem 2 is valid.

Theorem 3. Let $S$ be a hyperplane with a unit normal vector $v \in \mathbb{R}^{d}$ and $(\psi(t, x))_{t \geq 0, x \in S}$ be a continuous function satisfying the following inequality $|\psi(t, x)| \leq C_{T} t^{-\beta}, 0<t \leq T, x \in S$ with some constants $\beta<1$ and $C_{T}>0$ (the last one can depend on $T$ ) for each $T>0$. Then for all $t>0, x \in S$ the following relations

$$
\lim _{y \rightarrow x \pm} \mathbf{B}_{v} v(t, \cdot)(y)=\mp \psi(t, x)
$$

hold true, where $y \rightarrow x+$, (or $y \rightarrow x-$ ) means that $y \rightarrow z$ in the way that $(y-x, v)>0$ (or $(y-x, v)<0)$.

Proof. The proof of this theorem repeats the proof of Theorem 2 with some simplification.

Acknowledgment. The authors thank to Prof. Mykola Portenko for useful discussions on the topic of the paper and an anonymous referee for valuable comments that helped to improve the article. 


\section{REFERENCES}

[1] Eidelman S. D., Ivasyshen S. D., Kochubei A. N. Analytic methods in the theory of differential and pseudodifferential equations of parabolic type. In: Ball J.A., Böttcher A., Dym H., Langer H., Tretter C. (Eds.) Operator Theory: Advances and Applications, 152. Birkhäuser, Basel, 2004. doi:10.1007/978-3-0348-7844-9

[2] Fridman A. A. Partial differential equations of parabolic type. Prentige-Hall Inc., Englewood Cliffs, N. J., 1964.

[3] Osypchuk M. M., Portenko M. I. On simple-layer potentials for one class of pseudo-differential equations. Ukrainian Math. J. 2016, 67 (11), 1704-1720. doi:10.1007/s11253-016-1184-7 (translation of Ukrainian Math. J. 2015, 67 (11), 1512-1524. (in Ukrainian))

Received 25.05.2019

Revised 28.11.2019

Мамалига Х.В., Осипчук М.М. Потенціали простого шару для одного класу псевдодиференціальних рівнянь пов'язаних з лінійними перетвореннями симетричного $\alpha$-стійкого випадкового процесу // Карпатські матем. публ. - 2019. - Т.11, №2. - С. 350-360.

Стаття присвячена дослідженню невиродженого лінійного перетворення симетричного $\alpha$ стійкого випадкового процесу в евклідовому просторі $\mathbb{R}^{d}$. Результат цього перетворення $\epsilon$ процесом Маркова в $\mathbb{R}^{d}$, чий твірний оператор задається символом $\left(-(Q \xi, \xi)^{\alpha / 2}\right)_{\xi \in \mathbb{R}^{d}} 3$ деякою симетричною додатно визначеною $d \times d$-матрицею $Q$ та фріксованим $\alpha \in(1,2)$. Щільність ймовірності переходу цього процесу $є$ фундаментальним розв'язком деякого параболічного псевдодиференціального рівняння. Вводиться поняття потенціалу простого шару та досліджуються його властивості. Зокрема встановлено оператор, який відіграє роль градієнта в класичній теорії. Аоведено аналог класичної теореми про стрибок конормальної похідної потенціалу простого шару. Ця властивість потенціалу простого шалу може бути використана для побудови розв'язків деяких крайових задач для розглянутих параболічних псевдодиференціальних рівнянь. Якшо $\alpha=2$, розглянутий процес $є$ лінійним перетворенням процесу броунівського руху і всі досліджені властивості потенціалу простого шару добре відомі.

Ключові слова і фрази: псевдодиференціальне рівняння, потенціал простого шару, $\alpha$-стійкий випадковий процес, теорема про стрибок. 